\title{
A Low-Cost Unmanned Underwater Vehicle Prototype for Shallow Water Tasks
}

\author{
W.H. Wang, Member, IEEE, X.Q. Chen, Senior Member, IEEE, A. Marburg, J.G. Chase, C. Hann
}

\begin{abstract}
Unmanned underwater vehicles (UUVs) have received worldwide attention and been widely used in various applications. In this paper, a UUV prototype recently developed at the University of Canterbury is introduced, featuring low cost and ready use for shallow water tasks, especially for inspecting and cleaning sea chests of ships for biosecurity purpose. The main hull of the UUV is made of PVC, with a dimension of $400 \mathrm{~mm}$ in diameter and $800 \mathrm{~mm}$ in length. External frames are constructed for mounting two horizontal propellers, four vertical thrusters, and onboard batteries. The maximum thrust force up to $10 \mathrm{~kg}$ provided by the propellers can generate a forward/backward speed of up to $1.4 \mathrm{~m} / \mathrm{s}$ for the $120 \mathrm{~kg}$ UUV. The vertical thrusters provide depth control with a full load of $20 \mathrm{~kg}$. The UUV is equipped with a range of sensors capable of sensing its instantaneous temperature, depth, attitude and surrounding environments. Overall, the UUV costs less than US\$10,000 and provides an excellent platform for further underwater vehicle development targeting shallow water tasks with a working depth up to $20 \mathrm{~m}$.
\end{abstract}

Index Terms-Unmanned underwater vehicle, low cost, hull design, shallow water inspection

\section{INTRODUCTION}

Unmanned underwater vehicles (UUVs) are devices used to perform tasks submerged in water. Although many names are given to the devices, such as remotely operated vehicles (ROVs), submersible devices, or remote controlled submarines, to name just a few, the fundamental task for these devices is fairly well defined. They operate in conditions and perform task that humans cannot or are not efficient enough [].

UUVs have been developed since the 1960s, driven by the demand from the US Navy [], which required them to perform deep sea rescue and salvage operations. Since then, other sectors have realized the potential of such devices for all manner of tasks. The first of these was the oil and gas industry []. These companies employed UUVs to reinforce in the development of off shore oil fields []. In the 1980's, UUVs came into a new era as they were able to operate at depths well below commercial diver limits []. Falling oil prices and a global recession resulted in a stagnant period in terms of UUV development in the mid 1980s. Since then however, UUVs have been developing at a fast rate [].

UUVs are now being used in a wide range of applications, such as to locate historic ship wrecks [] like the Titanic, to map the sea floor [], to detect objects [1], to secure harbours

Wang, Chen, Chase, and Hann are with the Dept. of Mechanical Engineering, University of Canterbury, Private Bag 4800, Christchurch 8140, New Zealand (e-mail: wenhui.wang@ canterbury.ac.nz).

Marburg is with Geospatial Research Centre (NZ) Ltd, Private Bag 4800, Christchurch 8140, New Zealand

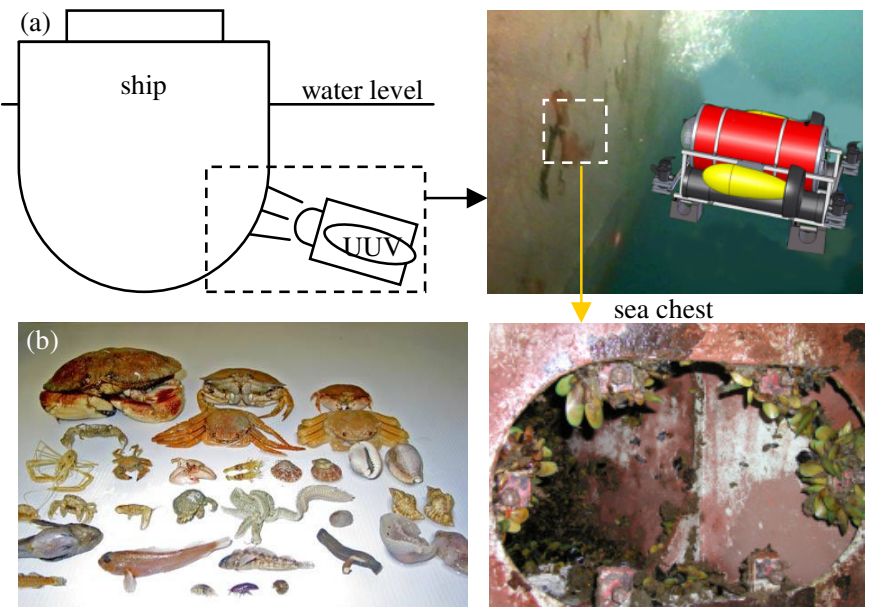

Fig. 1. Unmanned underwater vehicle inspecting and cleaning sea chest of ships. (a) The diagram of the UUV working on the sea chest of the ship. (b) A range of foreign invaders hiding in the sea chest.

[], to search for seamines [] and most recently in scientific applications [][].... As more than half of our oceans are deeper then $3 \mathrm{~km}$, one direction of the UUV developments is to explore deep waters. However, development of such UUVs imposes extreme stringent on hardware [], incurring an unaffordable cost to most of the labs. By contrast, UUVs for shallow waters recently have gained more attention because of its wide use and affordable cost. At the University of Canterbury, a UUV prototype has been recently designed with the primary purpose for inspecting and cleaning sea chests of ships (Fig. 1a).

Sea chests are the intake areas in the hulls of ships for seawater used for ballast, engine-cooling and fire-fighting. Grates on the outside of the chests prevent large organisms from being entrained in the water but many smaller organisms (Fig. 1b) survive in the sea chests and are transported around the world creating a biosecurity risk. It is highly prioritized in New Zealand to underpin the development of systems and tools to protect native flora and fauna against invasion by unwanted foreign organisms.

To take right actions on the foreign organisms, the first task is to inspect the sea chests and collect information about the invaders in the specific regions. Currently, divers are sent to do the job, which has inherent problems, including i) high cost, ii) unavailability, iii) safety concerns, iv) low throughput, and v) unsustainable working time. To reduce the working load of divers and to accelerate the treatment significantly, it is highly desirable to deploy affordable UUVs 
to inspect and clean the sea chests. In this paper, therefore, a low cost UUV prototype is introduced, with the emphasis on design. The control and navigation part of the UUV is being implemented and thus not covered. The design of the UUV is primarily purposed for this application where the working depth is less than $20 \mathrm{~m}$. However, the UUV is readily reconfigurable for other shallow water applications.

Section II deals with the vehicle hull design firstly. Then, Section III presents the thrusters that provide propulsion and steering for the UUV. Next, Section IV discusses the design for ballast and depth control. Finally, Section V covers the electronics and control structure for the UUV. The paper ends with a summary conclusion.

\section{Hull Design}

Fig. 2 shows the UUV prototype, which consists of basic components, including main hull, two horizontal propellers, four vertical thrusters, two batteries, an external frame, and electronics inside the main hull. This section focuses on the hull design.

There are a number of different ways in which hull design can be tackled []. These different design methods are in most cases specific to the submarines situation/task. In spite of methods, the main hull must be able to accomplish a number of key challenges all at the same time in order to be suitable.

Aspects considered during the hull design included:

- Pressure and/or depth required

- Operating temperature ranges

- Structural integrity for additions and tapings

- Impact conditions

- Water permeability

- Visual appeal and aesthetics

- Accessibility

- Versatility

- Practicality

- Restrictions for future additions

- Size requirements

- Corrosion and chemical resistance

The foremost design decision is regarding the shape of the hull. Inspired by torpedoes and submarines, a cylindrical hull has been selected. A cylinder has favourable geometry for both pressure and dynamic reasons. A round hull has no stress concentrations. With appropriate nose and tail cones, it can also exhibit a low drag along its main axis. To make the hull, three easily accessible materials are compared. The first option is to use a section of PVC storm water pipe. Such pipe is readily available. Another option involves having a hull made from a composite material such as carbon fibre or fibre glass. Mandrel spinning of such a hull will allow more freedom in radial dimensions. The process can in fact incorporate a varying radius along the length resulting in a slender, traditional hull. This process requires a large amount of design and set up time. A less desirable option is to use a section of metal pipe. This will have been cumbersome, expensive, and prone to corrosion. Based on the comparison, PVC storm water pipe option is chosen.

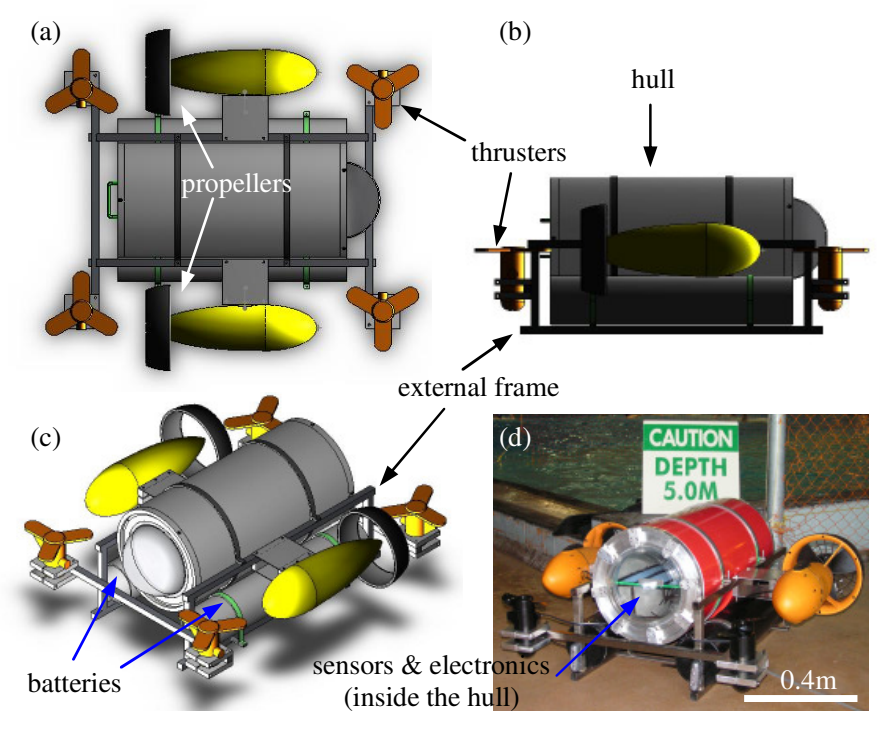

Fig. 2. The hull structure of the vehicle. (a)-(c) Design drawings of the vehicle: (a) Top view. (b) Side view. (c) Isometric view. (d) Real picture of the in-house made vehicle.

To complete the hull, two caps have been designed. These caps are attached to each end of the pipe in a manner that they reliably seal the hull. In addition to this, the caps allow access to the interior of the hull in such a manner that the device could be opened and closed out in the field - on a boat, or near the shore. To achieve good fluidic dynamics, the front cap has a dome shape.

The end cap design incorporates an aluminium ring that is permanently fixed to the hull and a removable aluminium plug. The plug fits snugly into the aluminium ring. Sealing is achieved with O-rings. Sealing directly to the PVC hull would have been more desirable however this is not an option due to two main reasons. Firstly, PVC does not provide a sealing surface as smooth and even as aluminium and is extremely hard to be machined due to the size of the pipe. Secondly, the PVC pipe is actually out of round. This would have further decreased sealing reliability.

Being one of the key elements of the UUV, the sealing arrangement is handled with special care. Its principle is based on self securing with the rising outside pressure in greater diving depth. This pressure presses on the hull as well as the end caps, which puts more tension on the sealing areas in both axial and radial directions.

The seal selected is a standard O-ring made of nitrile, being fresh and salt water resistant. This scheme is proved a reliable sealing.

\section{PROPUlsion AND SteERING}

The design incorporates 2 horizontal thrusters mounted on both sides of the UUV can provide movement both forward and backward as well as yaw by operating the thrusters in opposing directions. The thrusters are $12 \mathrm{~V}$ dive scooters that have a working depth of up to $20 \mathrm{~m}$.

The dive scooters are lightly modified to enable simple attachment to the external frame of the UUV. The thruster 


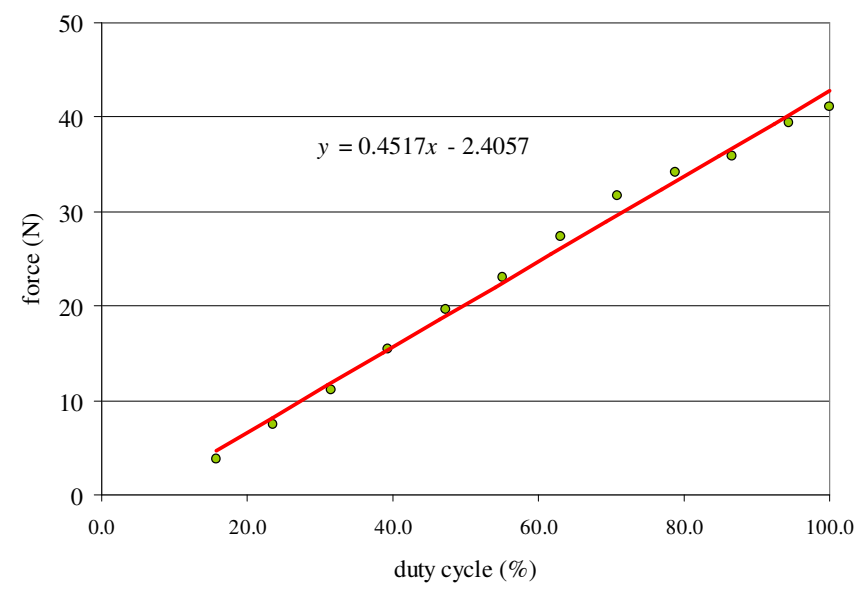

Fig. 3. Calibration of the motor: force with respect to duty cycle.

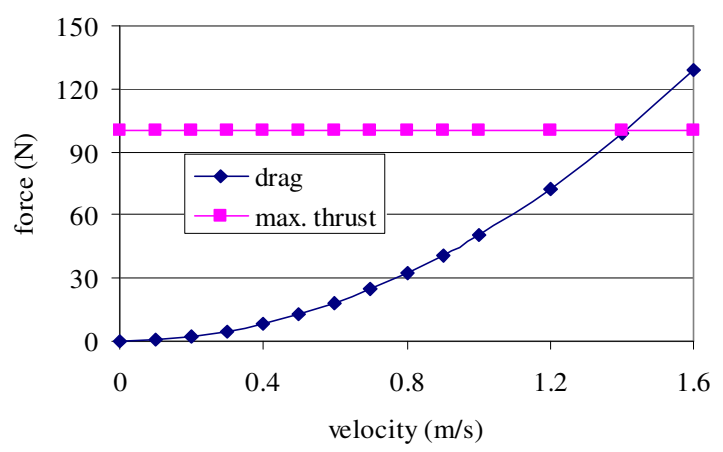

Fig. 4. Drag force of the UUV with different velocities.

mounts consist of two aluminium blocks which when bolted together clamp a plastic tab on each thruster. These provide a strong, secure mount which can be easily removed or adapted to other specifications.

The force that can be generated by the thruster is characterized, as shown in Fig. 3. A good linearity between the thruster force and the applied duty cycle can significantly facilitate the control scheme development in future.

A fluidic drag force model is established to evaluate the speed that the UUV can achieve. Fig. 4 shows the relationship between the drag force with respect to velocity of the vehicle. Under the full load of the two thrusters, the vehicle is able to achieve a forward or backward speed of up to $1.4 \mathrm{~m} / \mathrm{s}$.

\section{BALlast AND DePth CONTROL}

In the design, both static and dynamic diving principles are considered. Based on this, four initial concepts are developed: i) a piston type ballast tank, ii) a hydraulic pumping, iii) an air compressor system, and iv) thrust systems. The first three concepts belong to static diving technology, while the last concept employes the dynamic diving technology.

The piston ballast tank [] is one of the most common static diving methods applied in submarine modeling. A piston ballast tank consists of a cylinder and a movable piston, and it works as a big syringe. With one end of the cylinder connected to surrounding water, movement of the piston sucks water in or pushes it out. When water fills the tank, negative buoyancy is achieved, so the UUV starts to descend. Conversely, when the tank is emptied, the UUV is positively buoyant, so it ascends. This setup allows a control of pitch motions of the UUV. Moreover, the pistons can be moved by a linear actuator, which is electrically easy to control. Hence, accurate depth control can be achieved with proper programming.

A hydraulic pumping system [] is similar to the piston ballast tank, but using an internal reservoir of hydraulic fluid and a pump to actuate the piston's linear motion. Control of the valves and the pump for the hydraulic fluid allows it to flow in and out of the cylinders, so the surrounding water can be pumped in and out. Consequently, buoyancy of the UUV is changed.

An air compressor system [] is commonly used in a submarine. The system is composed of a storage tank of compressed air, a water tank and two valves that are normally closed. In order to descend, the vent valve is opened, so the pressure difference results in water flowing in from the opening in the bottom of the water tank. When a desired amount of water is obtained, the vent valve is closed. In order to force the water out, the blow valve is opened to allow the compressed air into the tank so the water is pushed out via the bottom opening. By letting the water in and out of the water tank, the buoyancy of the UUV is changed.

Thrusters are a dynamic diving method. They require the UUV to be always slightly positively buoyant, and then use the thrusters, vertically mounted, to force the UUV to dive, and hence to control the vertical motion of the UUV. This method consumes a lot of power, because in order to keep the UUV under water, thrusters must remain powered on all the time. Once the thrusters are off, positive buoyancy will be obtained, so the UUV starts ascending.

Selection of a suitable ballast system is dependent on various factors, such as design specifications, size and geometry of the UUV hull, depth required, and cost. In this design, the hull is made of a PVC pipe with an outer diameter of $400 \mathrm{~mm}$ and a length of $800 \mathrm{~mm}$; the required working depth is $20 \mathrm{~m}$, so the ballast system selected not only has to meet basic requirements, but also must be able to fit in the hull while at a relatively low cost.

Firstly, according to the geometry of the hull, the dimension of one cylindrical ballast tank is determined to be $160 \mathrm{~mm}$ inner diameter with a stroke length of $250 \mathrm{~mm}$. This is equivalent to approximately $5 \mathrm{~L}$ water storage. With two of these tanks installed in the UUV, a net force of $\pm 5 \mathrm{~kg}$ will be obtained. Additionally, the force required to actuate the piston head at $20 \mathrm{~m}$ is calculated to be around $6000 \mathrm{~N}$. To generate such force on the piston head, a powerful linear actuator is needed. The specific linear actuator (LA36 24V DC input, $6800 \mathrm{~N}$ max load, $250 \mathrm{~mm}$ stoke length) can be sourced from Linak Ltd in New Zealand. However, the linear actuator has a duty cycle of $20 \%$ at max load; that is, for every $20 \mathrm{~s}$ continuous work, it has to remain off for $80 \mathrm{~s}$ before operating again. Also, the cost of one linear actuator 


\section{is US\$1036.}

Secondly, the hydraulic pumping system can be customized from Scarlett Hydraulics Ltd, New Zealand. The overall system will be within a dimension of $500 \mathrm{~mm} \times$ $250 \mathrm{~mm} \times 250 \mathrm{~mm}$. It consists of a $1.2 \mathrm{KW}$ DC motor, a pump, a 4L hydraulic fluid tank, two dual solenoid valves and two cylindrical tanks. This system meets the required specifications; but it has some drawbacks. The system takes up much internal space of the hull, and it weighs approximate $20 \mathrm{~kg}$. In addition, the overall hydraulic pumping system will cost up to approximately US\$2264.

Next, the air compressor system is cost effective compared to piston ballast and hydraulic pumping system, and it is easy to operate by controlling the vent valve and the blow valve, yet the lack of accuracy in controlling compressed gas is a major disadvantage. However, the performance of the air compressor system is limited by the stored amount of gas. In this design, a 10L water tank will be needed to fulfil the changes in buoyancy. In other words, a gas cylinder containing 101 of air compressed to at least 3 bar is required for a single diving and rising cycle. Hence, in order to refill the gas cylinder, the UUV has to float to the waters surface before all the air runs out. Regarding the on-site requirement, that the UUV should operate for hours, the air tank must either be much bigger or higher pressurized, which leads to security issues.

Finally, using thrusters are a dynamic diving approach. They are different from the previous three systems since all three systems had to be installed inside the UUV, whereas the thrusters can be assembled externally. Hence, sealing is not as critical as the other concepts that can cause damage to internal electronic components if leakage occurred. Additionally, the thrusters can be sourced from Pu Tuo Hai Qiang Ltd, Zhou Shan, China for US\$55/unit. Each thruster will fit in a box of $215 \mathrm{~mm} \times 215 \mathrm{~mm} \times 80 \mathrm{~mm}$, and are driven by a $12 \mathrm{~V} \mathrm{DC}$ motor with a max thrust force of $5 \mathrm{~kg}$ under water. By mounting the desired number of thrusters, a wide range of motions can be controlled, such as pitch and roll control. Each concept has its own advantages and disadvantages, comparison between these options is summarized in Table I.

In this design, the major driving factors for the selection of ballast system are the cost and reliability. Piston ballast tank and thrusters systems are reliable since these two depth control methods have been widely employed in most unmanned underwater vehicle development. But considering the cost, the thruster system is more cost effective. Hence, the thruster system is chosen as the final design.

There are four thrusters vertically mounted around the UUV with one at each corner (See Fig. 2). Mounting four thrusters on the UUV produces a total of $20 \mathrm{~kg}$ thrust force at full load, and allows a wide range of motion control. They are able to control not only the vertical up and down motion, but also the pitch and roll motion. To achieve this, each of the thrusters is connected to a speed control module that can be controlled via a central microprocessor. By inputting different digital signals, various speeds are generated. As a result, a wide range of forces can be produced by each of the thrusters. Therefore, desired motion control can be obtained by different combinations.

\section{ELECTRONICS AND CONTROL}

\section{A. Power Supply}

In the design, although at this stage, the control module has not been achieved for the UUV, with the long-term goal for the UUV to become completely autonomous the power supply had to be located onboard, unlike many current models which take power over an umbilical link []. Since all the systems onboard the UUV are electric, sealed lead acid batteries are chosen for the power supply. These batteries have high capacity and can deliver higher currents, than other types of rechargeable battery. They are stable, mechanically robust and can work in any orientation, important considerations in a device of this type. In order to supply enough current for the entire machine several batteries have to be joined together. Instead of adding dead weight to achieve neutral buoyancy extra batteries can be added so that the running time of the UUV is higher than that required.

It is desired to have battery compartments separate from the main hull so that they can be interchanged in the field without having to open the main hull. To accommodate this two tubes have been fitted below the hull to house batteries. Within these tubes are the batteries connected to two bus bars. Each battery is fused prior to connecting to the bus bar, and the bars are isolated to the greatest extent possible to increase safety. These bus bars are then wired into the main hull; a waterproof socket needs to be added in the future to enable the quick interchange of battery compartments. A similar bus system exists inside the hull with connections to motors and electronic power supplies. Each of these internal connections is similarly fused. It is desired to have the bus intelligently monitored so that the state of each battery can be accessed and faults found.

\section{B. Central Processing Unit}

The central processing unit is responsible for accessing sensors, processing data and setting control outputs such as motor speeds.

Several systems are considered for this unit, an embedded system using microprocessors, FPGAs or a small desktop PC. A microprocessor system, most likely based on an ARM processor would have low cost, size and power requirements and is easy to interface to both analogue and digital sensors, motors and other actuators. The processing power and memory allocations of these microprocessors are more than sufficient for the simple control tasks, but will struggle with further development such as image processing. An FPGA system would also be small and have low power requirements, but would be more expensive than the microprocessor system. FPGAs work very well for fast, complex processing tasks such as image processing. However due to the complexity of programming these devices, they should be used in parallel with another system. The last system considered is a small desktop PC (Fig. 5). Although a desktop PC is bigger, more 
TABLE I

BALLAST COMPARISON

\begin{tabular}{|c|c|c|c|c|c|c|}
\hline & Diving Tech & Installation & Buoyancy & Sealing & Reliability & $\begin{array}{l}\text { Overall Cost }{ }^{\dagger} \\
\text { (US\$) }\end{array}$ \\
\hline Piston ballast tanks & Static & Internal & +ve, -ve, Neutral & Difficult & Used in most remote submarines & 2500 \\
\hline Hydraulic pumping system & Static & Internal & +ve, -ve, Neutral & Difficult & Not reliable & 2710 \\
\hline Air compressor & Static & Internal & +ve, -ve, Neutral & Difficult & $\begin{array}{l}\text { Air on board is limited, com- } \\
\text { pressed air hard to handle }\end{array}$ & 420 \\
\hline Thrusters & Dynamic & External & $+\mathrm{ve}$ & None & Used in most ROVs with big size & 500 \\
\hline
\end{tabular}

${ }^{\dagger}$ The cost is estimated as an overall system.

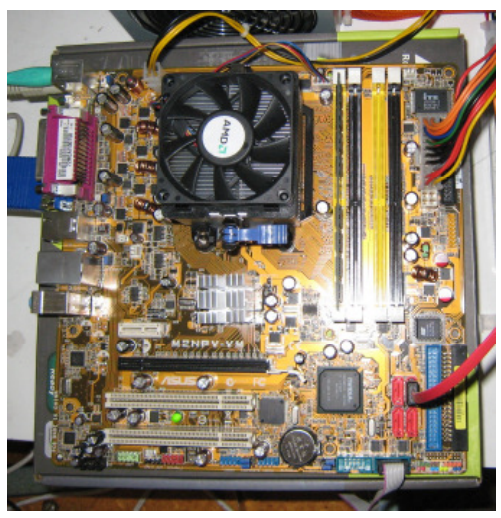

Fig. 5. On board computer.

expensive and consume more power than either of the above options, it provides immense processing power, memory and a diverse range of peripherals. It is chosen for the following main reasons:

- Added power requirements were not an issue since we have a sizeable power supplies.

- Processing power is more than adequate for this and future years development.

- Large volumes of memory are available, both volatile for program execution and solid state for storage of gathered data.

- Despite not having direct access to sensors and control units, the diverse range of peripherals available can be used, including USB, RS232 and Ethernet.

- A USB module is already provided for the webcam and an Ethernet module is provided for remote connection.

- The technical supervisor was prepared to spend the extra money to allow for future expansion.

An AMD Sempron 3000+ processor and ASUS M2NPV motherboard are used for this purpose. These models are chosen as they have lower power requirements and heat generation than other models. Software has been written to interface the central processing unit with sensors, motor controllers and a remote control PC. An automotive power supply (Mini-Box) is used to provide power for the computer; this power supply takes a $12 \mathrm{~V}$ DC input and converts it to the ATX standard power supply required by the PC. This module is designed to be used in an electrically noisy and hostile environment and is ideally suited to our purpose.

\section{Sensors}

When the UUV becomes autonomous there will be a large and extensive sensor suite onboard. Currently, there are sensors onboard to measure

- water pressure, from which depth can be determined;

- water temperature, inner hull temperature and humidity;

- the UUV position in the three principal axes: yaw, pitch and roll; and

- visual feedback.

Submersible pressure sensors which are salt water tolerant and can measure up to the pressure required are difficult to acquire at low cost. Several can be found in the US with a cost greater than US\$754 each; eventually one was found at Mandeno Electronics for US\$121. This sensor can measure twice the depth required, and outputs an analogue output between 0 and $100 \mathrm{mV}$. Thermocouples were acquired from Farnell electronics to measure the water temperature; these also have an analogue output, relevant to the temperature difference between the two ends of the thermocouple. TMP100 sensors (Texas Instruments) are used to measure the base temperature of the thermocouple, and the hulls interior temperature. These sensors give a digital output using the I2C protocol. A HF3223 humidity sensor (Digi-Key) is used to measure humidity inside the hull. A MMA7260QT accelerometer (Freescale Semiconductor) is used to calculate orientation. The accelerometer has a $0-2.5 \mathrm{~V}$ analogue output. The connection of the sensors is shown in Fig. 6.

To eliminate signal noise, An Atmel AT90USB82 microprocessor is connected to the USB ports of the computer to move all noise sensitive data to the acquisition points. The analogue sensors are amplified using an INA2322 instrumentation amplifier, if necessary and read by an ADS7828 analogue to digital converter. This converter is then connected to the Atmel microprocessor using a common I2C bus with the TMP100. The humidity sensor is attached to a clock input which converts the frequency based signal to a humidity based reading. The microprocessor performs some basic processing on this data, temperature compensating the pressure sensor and thermocouple and calculating yaw, pitch and roll from the accelerometer readings.

A visual sensor is included through a Logitech webcam, this connects directly to the computers USB port and feed the video stream back over the network. At this stage no image processing is done on this stream and is included purely to assist in manual control of the UUV. 


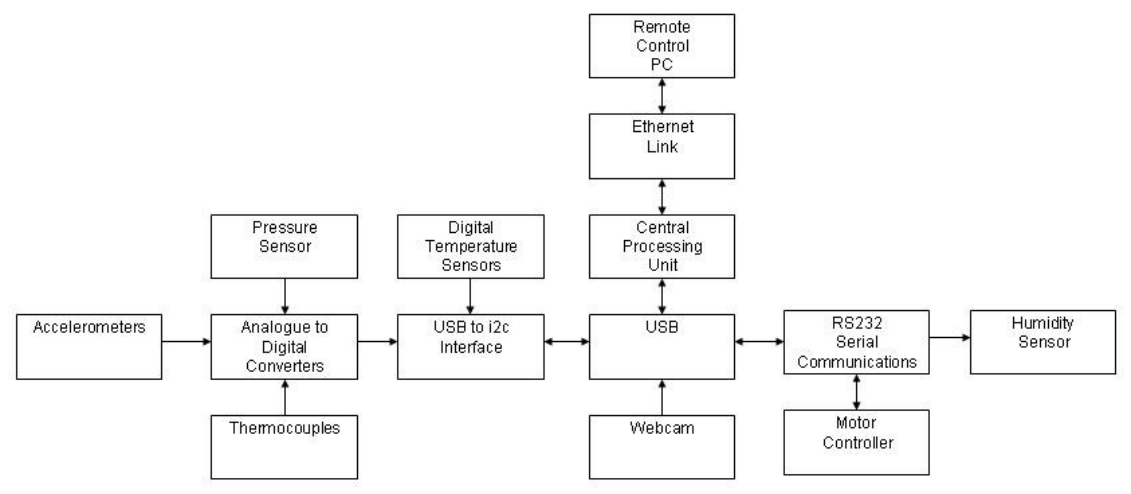

Fig. 6. The block diagram for electronic systems and control.

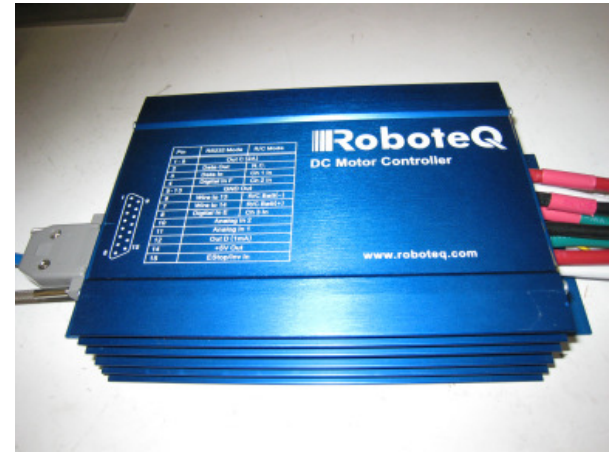

Fig. 7. The controller for both horizontal and vertical thrusters.

\section{Propulsion Motor Driver}

For the six motors (two for horizontal propulsion and four for vertical ballast control), three RoboteQ AX2500 (Fig. 7) motor controllers are used for control. Each controller is able to control two motors up to $120 \mathrm{amps}$, much higher than the 25 amps needed by the motors in use. The controllers are controlled via an RS232 (serial port) interface, which are already available on the computer motherboard. Computer control of the controllers is easily achieved through a LabView or MATLAB interface, either manually or automatically.

\section{E. Control System and Communications}

During testing and development, remote control is required for the UUV. Sensors readings need to be sent to a user, and control signals sent back to the UUV. A video feed from the webcam is also desired to be displayed to provide the operator with visual feedback. High frequency radio transmissions are impossible underwater due to the high losses encountered during the air/water boundary []. Lower frequency transmissions could have been used to communicate with the UUV, but they do not possess enough bandwidth to send the required data. An umbilical ethernet cable is being used for this remote link between the UUV and an external control computer. Fig. 6 shows the electronics and control structure.

\section{CONCLUSION}

For shallow water tasks, such as to inspect and clean sea chests of ships, a UUV has been successfully prototyped at the University of Canterbury. It features low cost and wide use for normal shallow water tasks with a working depth up to $20 \mathrm{~m}$, and a forward/backward speed up to $1.4 \mathrm{~m} / \mathrm{s}$. Each part of the UUV is deliberately chosen based on a comparison of available options when possible. The prototype has a complete set of components including vehicle hull, propulsion, depth control, sensors and electronics, batteries, and communications, at a total cost less than US $\$ 10,000$. With these elements, the control of the UUV is possible and being implemented. The overall underwater vehicle will be a good platform for research purposes, as well as for its applied purposes including the inspecting and cleaning sea chests of ships for less labor-intensive biosecurity checking at an affordable cost.

\section{REFERENCES}

[1] H. Kondoa and T. Ura, "Navigation of an AUV for investigation of underwater structures," Control Engineering Practice, vol. 12, p. 1551C1559, 2004. 